\title{
Effect of diuretics on plasma aldosterone and potassium in primary hypertension: A systematic review and meta-analysis
}

\author{
Ryan McNally ${ }^{1}$, Bushra Farukh ${ }^{1}$, P J Chowienczyk ${ }^{2}$, and Luca Faconti ${ }^{3}$ \\ ${ }^{1}$ King's College London \\ ${ }^{2}$ Affiliation not available \\ ${ }^{3}$ King's College London British Heart Foundation Centre, School of Cardiovascular \\ Medicine and Sciences, Department of Clinical Pharmacology
}

June 1,2021

\begin{abstract}
Aim: Different to inhibitory drugs of the renin-angiotensin-aldosterone system (RAAS), diuretics are known to decrease blood pressure (BP) and stimulate renin release by the kidneys. Despite plasma aldosterone (PA) level is mostly regulated by the RAAS activity, serum potassium has been shown to be an important factor in animal models and humans. Here we perform a systematic review and meta-analysis of randomized-controlled trials investigating the effects of diuretic therapy on PA and its correlation with change in potassium and BP. Methods: Three databases were searched: MEDLINE, EMBASE and The Cochrane Central Register of Controlled Trials (CENTRAL). Titles were firstly screened by title and abstract for relevancy before full-text articles were assessed for eligibility according to a pre-defined inclusion/exclusion criteria. Results: A total of 1139 articles were retrieved of which 45 met the pre-specified inclusion/exclusion criteria. The average standardised difference in mean PA change was similar for all classes of diuretic (mean, 95\% CI); thiazide/thiazide-like 0.304 (0.169, 0.440), loop 0.927 $(0.37,1.49), \mathrm{MRA} /$ potassium-sparing $0.264(0.174,0.355)$ and combination $0.466(0.142,0.789), \mathrm{Q}=6.475, \mathrm{P}=0.091$. In subjects previously untreated with another antihypertensive, there was a significant relationship between PA change and change in systolic BP but no relationship with the change in potassium. Conclusion: In RCTs of diuretic therapy in hypertension, there is an increase in PA with all classes of diuretic and no between-class heterogeneity. Change in PA is not related with potassium but correlates to the change in BP in subjects previously untreated with another antihypertensive medication.
\end{abstract}

\section{INTRODUCTION}

The renin-angiotensin-aldosterone system (RAAS) is a complex neuroendocrine system that regulates salt and water homeostasis, blood pressure (BP) and tissue proliferation amongst several other actions [1]. Blockers of RAAS are a cornerstone in the treatment of hypertension working at least in part by reducing the formation or blocking the effects of angiotensin II and plasma aldosterone (PA) which is recognized as an independent cardiovascular risk factor promoting cardiovascular and renal inflammation, fibrosis, and remodelling [2].

Compared to RAAS inhibitory drugs, diuretics have more complex mechanism of action [3] which includes an initial reduction in plasma volume and a sustained decline in peripheral resistance, thereby improving the underlying haemodynamic defect of hypertension [4-7]. Under acute and chronic conditions, diuretics induce an increase in plasma renin activity (PRA) [8] but whether diuretics also increases PA has been debated [9] . In fact, apart from the level of activation of the RAAS; potassium [10-12] (which is also affected by diuretic treatment) plays an important role in the regulation of PA production and some diuretics (such as mineralocorticoid receptor antagonists) are known to have direct inhibitory action on aldosterone formation.

Therefore, the main objective of the present study was to perform a systematic review and meta-analysis of 
randomised clinical trials (RCT) where diuretics were used to treat hypertension and measurements of PA were available before and after diuretic treatment to address if they lead to a sustained increased in PA. The secondary outcomes were to establish (a) if there is correlation between difference in PA and plasma potassium between diuretic classes (b) if the decrease in BP relates to the difference in PA.

\section{METHODS}

\section{Search strategy}

This systematic review and meta-analysis was carried out in accordance with PRISMA (Preferred Reporting Items for Systematic Reviews and Meta-Analyses) guidelines [13]. A systematic literature search was performed on three databases; Ovid MEDLINE(R) Epub Ahead of Print, In-Process \& Other Non-Indexed Citations, Ovid MEDLINE(R) Daily and Ovid MEDLINE(R), (1946 to $16^{\text {th }}$ September 2020), EMBASE (1974 to $16^{\text {th }}$ September 2020) and The Cochrane Central Register of Controlled Trials (CENTRAL) databases (up to $16^{\text {th }}$ September 2020). Studies which were included were trials of diuretics used either as mono- or multi-therapy, which examined how they affected plasma aldosterone $+/$ - renin, serum potassium and blood pressure. The keywords used included 'thiazide', 'thiazide-like', 'potassium-sparing', and 'diuretic'. Medical subject headings (MeSH) and non-MeSH terms were used to search the databases for relevant publications. The full search strategy for MEDLINE is provided in the supplementary material.

\section{Study selection and eligibility criteria}

Papers were initially screened by title and abstract. Studies were eligible for inclusion if they were a randomized-controlled trial (RCT) performed in hypertensive human subjects [?]18 years old, examining antihypertensive effects of either a thiazide, thiazide-like, loop, mineralocorticoid receptor antagonist (MRA) or potassium-sparing diuretic with a duration of at least one week. Studies investigating novel diuretics not licensed for clinical use were excluded as were studies in which subjects had pulmonary arterial hypertension or heart failure. All studies were required to have examined PA with results available before and during diuretic treatment or, in the case of placebo-controlled trials, during treatment with placebo. Studies were eligible if diuretic therapy was added to either no previous therapy or to stable background treatment. The search was limited to the English language only and review articles were disregarded. Titles and abstracts were screened by one author (RJM), and the same author reviewed the full-text articles.

\section{Data collection process}

Data were extracted independently by one author (RJM) using a standard form. This included: author, year of publication, class of diuretic(s) and dose used, protocol (including presence of background therapy and whether placebo controlled), sample size, average age, sex distribution, ethnicity (if available) and prevalence of diabetes (if available). For the outcome measurements, mean ( \pm standard deviation/standard error) of values for $\mathrm{BP}$ and aldosterone before and during diuretic (and before and during placebo treatment in placebo-controlled studies) and the difference between values on and before treatment. Where available, the difference in renin, serum potassium and systolic blood pressure (SBP) were extracted. If standard deviations were not reported these were calculated from standard errors, P-values or from confidence intervals. The duration of diuretic treatment at the time of measurement was also recorded. Where only graphical reports of measurements were available, an estimation from the graph was taken if it was judged to be accurate to within 10\%. Units of aldosterone were converted to pmol/L for analysis if other units were used.

\section{Quantitative data synthesis and statistical analysis}

Meta-analysis was conducted using Comprehensive Meta-Analysis Software Version 3 (Biostat, Englewood, New Jersey) [14]. Net changes in PA, renin, potassium and systolic blood pressure were obtained as the difference from baseline after treatment with either diuretic or placebo. If there was no standard error of the mean change stated, it was estimated from the p-value, number of observations and size of the change. A random-effects model was used to compensate for between-study heterogeneity in terms of demographic inconsistencies and different diuretic doses [15] with calculation of the standardised mean difference in PA 
and its $95 \%$ confidence interval (CI). Raw mean differences were calculated for plasma potassium, and SBP and standardised differences for PRA. Statistical heterogeneity was assessed using Cochran's Q test [16]. $P$ $<0.05$ was considered statistically significant and all tests were 2 -tailed.

\section{Meta-regression}

Random-effects meta-regression was performed using the method of moments to evaluate the association between standardized difference in PA and change in SBP in the overall data and in subjects previously untreated with another antihypertensive. The same method was used to establish the association between aldosterone change and change in serum potassium.

\section{Publication bias}

Potential publication bias was assessed by inspection of Begg's funnel plot asymmetry and Egger's asymmetry tests [17].

\section{RESULTS}

\section{Description of studies}

The study selection process is detailed in a flow chart as per PRISMA guidelines (Figure 1). The initial MEDLINE search returned 191 results, Embase 384 and Central 564 (1139 in total). After removal of duplicates there were 967 articles of which 769 were excluded based on title and abstract. The remaining 198 full-text articles were assessed for eligibility and 153 were excluded for various reasons (Figure 1). The remaining 45 articles were included in the qualitative synthesis. MRA and potassium-sparing diuretics were grouped together for the analysis as only one study had measurements for a potassium-sparing diuretic (amiloride) with the remainder MRAs. The most commonly used diuretic class was the MRA/potassium-sparing class. Classes of diuretic were thiazide/thiazide-like (24/45, 53\%), MRA/potassium-sparing (28/45, 62\%), loop $(4 / 45,9 \%)$ and combination $(3 / 45,7 \%)$. Individual diuretics were hydrochlorothiazide, amiloride, indapamide, bendroflumethiazide, chlorthalidone, canrenone, furosemide, spironolactone, eplerenone, tielinic acid and torsemide. Details of individual trials are summarised in table 1. In those studies where patients where previously treated with another antihypertensive medication before the diuretic was added, an ACE/ARB was included in over 50\%. For renin sub-analysis, plasma renin activity (PRA) was used as this was the most common measure of renin in the studies.

\section{Primary Outcome Meta-analysis: Effect of placebo and diuretic on plasma aldosterone}

Placebo had a negligible effect on PA in the present analysis (Fig 2D). Standardized difference in mean PA after placebo was $-0.11(95 \%$ CI $-0.36,0.14)$. With diuretic therapy, all diuretic classes led to a significant increase in PA but there was no between-class heterogeneity (Fig 2A). The average standardised difference in mean PA change was: thiazide/thiazide-like $0.304(0.169,0.440)$, loop $0.927(0.37,1.49), \mathrm{MRA} /$ potassiumsparing $0.264(0.174,0.355)$ and combination $0.466(0.142,0.789), \mathrm{Q}=6.475, \mathrm{P}=0.091$. In studies where there was no background antihypertensive use/a washout, the average increase in PA was $0.392(0.234$, 0.550 , Figure $2 \mathrm{~B}$ ). There was a similar, increase in PA for those in whom diuretic was added to previous antihypertensives: 0.300 (0.046, 0.553, Figure 2C). After separating MRA from potassium-sparing diuretics, the same homogeneity between classes was found.

\section{Secondary Outcomes Meta-analysis: Effect of placebo and diuretic on serum potassium, plasma renin activity and blood pressure}

Changes in serum potassium were: thiazide/thiazide-like $-0.275(-0.62,-0.78) \mathrm{mEq} / \mathrm{L}$, loop -0.617 (-0.983, $0.251) \mathrm{mEq} / \mathrm{L}, \mathrm{K}+$ sparing/MRA $0.248(0.151,0.345) \mathrm{mEq} / \mathrm{L}$ and combination $0.048(-0.327,0.422) \mathrm{mEq} / \mathrm{L}$, with significant between-class heterogeneity $(\mathrm{Q}=49.4, \mathrm{P}<0.001$, Figure 3$)$. The analysis of PRA and SBP can be found in the supplementary material. Of the 45 included studies, 26 had differences in SBP, 26 had differences in serum potassium and 23 had differences in PRA both before and after diuretic. All diuretics decreased blood pressure significantly, apart from the loop diuretics, with combination diuretics lowering SBP by the largest amount $(-23.4[-35.33,-11.48] \mathrm{mmHg})$. In studies where there was no background 
antihypertensive use/a washout, the average decrease in SBP was $-13.05(-17.7,-8.40) \mathrm{mmHg}$ and similar those in whom diuretic was added to previous antihypertensives: $-14.76(-18.78,-10.75) \mathrm{mmHg}$.

The overall average increase standardised mean PRA difference was $0.560(0.275,0.846)$ with a similar trend in both previously untreated subjects and those receiving another antihypertensive medication.

Meta-regression: Relation of change in serum potassium after diuretic to change in aldosterone

Random-effects meta-regression was also used to examine whether change in PA was associated with serum potassium and we found there was no relationship (coefficient $-0.09,95 \% \mathrm{CI}-0.30,0.12, \mathrm{P}=0.402$, Figure 4A) with similar findings in studies where studies were the participants were previously untreated/washout (coefficient -0.13, 95\% CI -0.31, 0.06, $\mathrm{P}=0.174$, Figure 4B).

\section{Meta-regression: Relation of change in aldosterone after diuretic to change in SBP}

Random-effects meta-regression was performed to examine whether change in SBP was associated with change in PA. In the overall population, the changes in SBP were independent of changes in PA (coefficient $-0.005,95 \%$ CI $-0.018,0.009, \mathrm{P}=0.49$, Figure $5 \mathrm{~A}$ ). However, in studies were the participants were previously untreated/washout with another antihypertensive, there was a significant relationship between the change in PA and SBP (coefficient $-0.02,95 \%$ CI $-0.033,-0.01, \mathrm{P}<0.001$, Figure 5B).

\section{Publication bias}

The funnel plot of standard error vs. effect size was asymmetric and suggestive of potential publication bias. Presence of publication bias was also suggested by Egger's linear regression $(\mathrm{P}=<0.001)$. After adjustment of effect size for potential publication bias using the 'trim and fill' correction, fourteen potentially missing studies on the left side of the funnel plot were imputed leading to a corrected effect size that was slightly but not significantly less than the initial estimate (0.22 (95\% CI 0.14-0.29, Figure 6).

\section{DISCUSSION}

As far as we are aware, this is the first study to systematically review the effect of diuretic therapy on PA its correlation with potassium and BP change in hypertensive individuals. Diuretics have been used in treatment of hypertension for more than 50 years [18], both as monotherapy and in combination with other anti-hypertensive agents. Several investigations have confirmed that diuretic agents are safe, effective, well tolerated $[19,20]$ and can be considered as first line pharmacological agent in specific populations [21]. However, the short- and long-term mechanisms of action of the various classes of diuretics has been debated and it is known that chronic diuretic treatment leads to an increase in PRA suggesting activation of the RAAS. Wherever the raised PRA would also be accompanied by an increase in PA is still subject of debate and it is unclear if this relates to specific classes of diuretics and/or concomitant change of serum potassium which is an important regulator of aldosterone.

The main finding of the present analysis is that diuretics lead to an increase in PA, which doesn't differ between classes of diuretic, but which is significantly associated with change in SBP in previously untreated subjects. In the studies where PRA was also measured before and after treatment, a raise in PRA occurred (a finding in line with a previously published systematic review[22]) suggesting that increase in PRA could be driven by activation of the RAAS.

Whether the increased PA could be harmful is subject of debate and speculation on this topic are beyond the scope of this systematic review. It is however important to stress here that the RAAS is a complex system in which angiotensin II acts through two main receptor subtypes, the AT1 and the AT2 receptor. All classic physiological effects of angiotensin II, such as vasoconstriction, aldosterone production and water retention are largely mediated by the AT1 receptor which promotes hypertension, endothelial dysfunction, vascular remodelling and end organ damage. On the other hand, AT2 receptors elicits antithrombotic, ant inflammatory and natriuretic effects [23]. Thus, the activation of the RAAS could have complex actions according to the balance of the activation between the two types of receptors which counteract each other in their biological actions on the cardiovascular system [24,25]. 
Whilst there is evidence that PRA is helpful in selecting patients who will benefit from diuretic therapy [26], the potential use of change in PRA in guiding dose-titration and selecting class of diuretic remains speculative and was not confirmed by a recently published systematic review [27].

In that respect, a biomarker would be suitable for that use depending on whether a change can be detected in response to diuretic treatment and whether this relates to BP response. In our analysis the change in SBP with that of PA suggests that change in PA, as opposed to pre-treatment could be a useful marker to guide diuretic therapy (for example by increasing diuretic dose if there is inadequate rise in PA). To confirm this hypothesis, further studies are warranted. On the other hand it could be also speculated that the use of a concomitant medication might play a role in limiting the raise in PA which in turn could have a beneficial effects per se since it has been suggested that aldosterone could facilitate cardiac remodelling without affecting arterial pressure [28-30]. To elucidate this point, dedicated investigations would need to be designed and conducted.

We did not identify any correlation between change in serum potassium and PA. Apart from RAAS, the other major factor regulating aldosterone secretion is potassium. In man and in experimental animals, alterations in potassium balance as well as acute increments in serum potassium can stimulate aldosterone production. For example, in normal subjects, infusion of $10 \mathrm{mEq}$ of potassium produces a $25 \%$ increase in plasma aldosterone [10]. Changes in dietary potassium intake for as little as $24 \mathrm{hr}$ can also substantially modify the secretion of aldosterone from the adrenal glands induced by acute potassium administration: high dietary potassium intake enhances responsiveness, while low potassium intake reduces it [11]. Our results seem to suggest that variation of serum concentration of potassium per se might have a limited effect in regulating PA although there are suggestions that the mechanism could be relevant in specific populations [31].

Finally, the sub-analysis investigating MRA showed similar effects of these agents on PA compared to other diuretic classes. It has been reported that similarly to other inhibitory drugs of the RAAS [32,33], after an initial suppression/blockade of aldosterone, the PA level often returns to normal or even rises above pretreatment levels for the so-called escape mechanism $[34,35]$.

This review is subject to several limitations. We were unable to stratify results by ethnicity (since wellestablished difference in RAAS activity have been described) because the majority of studies were performed in Caucasians and in many studies, ethnicity was not reported. Studies in specific ethnic groups will be required to determine if effects of diuretics on PA differ according to ethnicity. The use of background therapy in some studies and a variable dose in others prevent a useful estimate of the effect size relating to a standard dose of diuretic. The duration of studies was relatively short and very few studies were performed with loop diuretics (which are not commonly used in hypertension). The MRA/potassium sparing group was mostly composed of spironolactone which in many trials was used at high dose unrepresentative of its current use in primary hypertension.

In conclusion, this systematic review and meta-analysis demonstrates that diuretic therapy in hypertension leads to an increase in PA which does not differ between classes of diuretics and which is related to the fall in SBP in previously untreated subjects .

\section{Author's contributions}

All authors developed the study concept and designed the research. RJM conducted the electronic searches, study selection and extraction. RJM \& LF performed data analysis with BF helping interpret the results. RJM, LF and PJC wrote the majority of the manuscript. All authors read and approved the final version of the manuscript.

\section{Conflict of interest}

The authors have no conflict of interest to declare.

\section{Funding sources}


Mr Ryan McNally is supported by an interdisciplinary studentship from the British Heart Foundation, King's BHF Centre of Research Excellence. The authors acknowledge financial support from the Department of Health via the National Institute for Health Research (NIHR) comprehensive Biomedical Research Centre and Clinical Research Facilities awards to Guy's and St Thomas' NHS Foundation Trust in partnership with King's College London and King's College Hospital NHS Foundation Trust.

\section{Acknowledgements: None}

\section{Disclosures}

None.

\section{REFEREN CES}

1. Atlas A. Steven. The Renin-Angiotensin Aldosterone System: Pathophysiological Role and Pharmacologic Inhibition. J Manag care Pharm. 2010;13:9-20.

2. White PC. Aldosterone: Direct effects on and production by the heart. J Clin Endocrinol Metab. $2003 ; 88: 2376-83$.

3. Kaplan NM. Diuretics as a basis of antihypertensive therapy: An overview. Drugs. 2000;59:21-5.

4. Brater DC. Pharmacology of Diuretics. Am J Med Sci. 2000;319:38-50.

5. Greenberg S, McGowan C, Xie J, Summer WR. Selective pulmonary and venous smooth muscle relaxation by furosemide: A comparison with morphine. J Pharmacol Exp Ther. 1994;270:1077-85.

6. Wiemer G, Fink E, Linz W, Hropot M, Scholkens BA, Wohlfart P. Furosemide enhances the release of endothelial kinins, nitric oxide and prostacyclin. J Pharmacol Exp Ther. 1994;271:1611-5.

7. Pickkers P, Hughes AD, Russel FGM, Thien T, Smits P. Thiazide-Induced Vasodilation in Humans Is Mediated by Potassium Channel Activation. 1998;

8. P. LIJNEN, R. FAGARD JS\& AA. EFFECT OF CHRONIC DIURETIC TREATMENT ON THE PLASMA RENIN-ANGIOTENSIN-ALDOSTERONE SYSTEM IN ESSENTIAL HYPERTENSION. Br J Clin Pharmacol. 1981;12:387-92.

9. Maxwell MH GC. Tricrynafen and hydrochlorothiazide in essential hypertension. Effect of the reninangiotensinaldosterone system and on electrolyte balance. In: A new class of diuretics with uricosuric activity. Postgrad Med Commun. 1978;5:43-50.

10. Himathongkam T, Dluhy RG WG. Potassim-aldosterone-renin interrelationships. J Clin Endocrinol Metab. 1975;41:153-9.

11. Williams GH, Tuck ML, Rose LI, Dluhy RG, Underwood RH. Studies of the control of plasma aldosterone concentration in normal man. 3. Response to sodium chloride infusion. J Clin Invest. 1972;51:2645-52.

12. EL B. Regulation of aldosterone secretion: current concepts and newer aspects. adv nephrol. 1977;7:10520 .

13. Moher D, Liberati A, Tetzlaff J, Altman DG. Preferred reporting items for systematic reviews and meta-analyses: the PRISMA statement. J Clin Epidemiol. 2009;62:1006-12.

14. Borenstein, M., Hedges, L., Higgins, J., \& Rothstein H. Comprehensive Meta-Analysis Version 3. Biostat, Englewood, NJ; 2013.

15. Sutton AJ, Abrams KR, Jones DR, Sheldon TA SF. Methods for meta-analysis in medical research. 2000.

16. Higgins JPT, Thomas J, Chandler J, Cumpston M, Li T, Page MJ WV, editor. Cochrane Handbook for Systematic Reviews of Interventions version 6.0 (updated July 2019). Cochrane; 2019. 
17. Egger M, Smith GD, Schneider M, Minder C. Bias in meta-analysis detected by a simple, graphical test. Br Med J. 1997;315:629-34.

18. Moser M, Feig PU. Fifty years of thiazide diuretic therapy for hypertension. Arch Intern Med. 2009;169:1851-6.

19. Roush GC, Sica DA. Diuretics for hypertension: A review and update. Am J Hypertens. 2016;29:1130-7.

20. Thomopoulos C, Parati G, Zanchetti A. Effects of blood pressure lowering on outcome incidence in hypertension.1. Overview, meta-analyses, and meta-regression analyses of randomized trials. J Hypertens. 2014;32:2285-95.

21. Wright JT Jr1, Dunn JK, Cutler JA, Davis BR, Cushman WC, Ford CE, Haywood LJ, Leenen FH, Margolis KL, Papademetriou V, Probstfield JL, Whelton PK HGACRG. Outcomes in hypertensive black and nonblack patients treated with chlorthalidone, amlodipine, and lisinopril. JAMA - J Am Med Assoc. 2005;293:1595-608.

22. McNally RJ, Faconti L, Cecelja M, Farukh B, Floyd CN, Chowienczyk PJ. Effect of diuretics on plasma renin activity in primary hypertension: A systematic review and meta-analysis. $\mathrm{Br} \mathrm{J}$ Clin Pharmacol. $2021 ; 2189-98$.

23. Carey RM. AT2 receptors: Potential therapeutic targets for hypertension. Am J Hypertens. 2017;30:33947.

24. Perrone MH, Deborah A, Perrone MH, Physiol C. renin-angiotensin; an-. 2020;

25. Stoll M, Steckelings UM, Paul M, Bottari SP, Metzger R, Unger T. The angiotensin AT2-receptor mediates inhibition of cell proliferation in coronary endothelial cells. J Clin Invest. 1995;95:651-7.

26. Furberg CD. Renin-guided treatment of hypertension: Time for action. Am J Hypertens [Internet]. Nature Publishing Group; 2010;23:929-30. Available from: http://dx.doi.org/10.1038/ajh.2010.135

27. McNally RJ, Morselli F, Farukh B, Chowienczyk PJ, Faconti L. A review of the prescribing trend of thiazide-type and thiazide-like diuretics in hypertension: A UK perspective. $\mathrm{Br} \mathrm{J}$ Clin Pharmacol. 2019;85:2707-13.

28. Weber KT, Sun Y, Guarda E. Structural Remodeling in Hypertensive Heart Disease and the Role of Hormones. 2011;

29. Young M, Dilley R, Funder J. Mineralocorticoids, hypertension, and cardiac fibrosis. J Clin Invest. $1965 ; 44: 1072-116$.

30. Atsuhisa Sato JF. High glucose stimulates aldosteronesterone-induced hypertrophy via type I mineralocorticoid receptors in neonatal rat cardiomyocytes.

31. Faconti L, McNally RJ, Farukh B, Adeyemi O, Cruickshank JK, Wilkinson IB, et al. Differences in hypertension phenotypes between Africans and Europeans: Role of environment. J Hypertens. 2020;38:127885.

32. Nussberger J, Brunner DB, Waeber B, Brunner HR. Specific measurement of angiotensin metabolites and in vitro generated angiotensin II in plasma. Hypertension. 1986;8:476-82.

33. Anton H. van den Meiracker, Peter J.J. Admiraal, Joop A. Janssen, Jan-Maarten Kroodsma, Wijnand A.M. de Ronde, Frans Boomsma, Joelle Sissmann, P.J. Blankestijn, Paul G.M. Mulder, Arie J. Man in 't Veld and MADHS. Hemodynamic and Biochemical Effects of the AT1 Receptor Antagonist Irbesartan in Hypertension. Hypertension. 1995;25:22-9.

34. Sawathiparnich P, Kumar S, Vaughan DE, Brown NJ. Spironolactone abolishes the relationship between aldosterone and plasminogen activator inhibitor-1 in humans. J Clin Endocrinol Metab. 2002;87:448-52. 
35. S Ichikawa, Y Tajima, T Sakamaki, H Matsuo, M Kogure, Y Hirano, S Yagi KM. Effect of spironolactone on fluid volumes and adrenal steroids in primary aldosteronism. Circulation. 1984;48:1184-96.

\section{FIGURE LEGENDS}

Figure 1 : PRISM diagram. Flow-chart of included \& excluded studies against a pre-defined search criteria.

Figure 2: Forest plots displaying standardized difference in mean PA and 95\% confidence intervals. A) overall, B) previously untreated with another antihypertensive, C) previously treated with another antihypertensive and D) placebo. Std diff, standardised difference; CI, confidence interval; MRA, mineralocorticoid receptor antagonists.

Figure 3: Forest plots displaying raw difference in mean plasma potassium and $95 \%$ confidence intervals. A) overall, B) previously untreated with another antihypertensive, C) previously treated with another antihypertensive and D) placebo. CI, confidence interval; MRA, mineralocorticoid receptor antagonists.

Figure 4: Meta-regression plot of the association between mean changes in PA with diuretic therapy and the change in serum potassium $(\mathrm{mEq} / \mathrm{L})$ in $(\mathrm{A})$ overall and $(\mathrm{B})$ previously untreated with another antihypertensive. Std diff, standardised difference.

Figure 5: Meta-regression plot of the association between mean changes in PA with diuretic therapy and the change in SBP in (A) overall and (B) previously untreated with another antihypertensive. SBP, systolic blood pressure;. Std diff, standardised difference.

Figure 6. Funnel plot displaying publication bias in the studies reporting the impact of diuretic therapy on PA change. Open diamond represents observed effect size; closed diamond represents imputed effect size. Std diff, standardised difference.

\begin{tabular}{|c|c|c|c|c|}
\hline Study ID, Year & Diuretic, dose & Study design, sample size & Age (years) & Male $(\%)$ \\
\hline Hood, 2007 & SPIRO 50-100mg & Crossover, 51 & $59.5 \pm 11.9$ & 54 \\
\hline Hood, 2007* & AMIL 20-40mg & Crossover, 51 & $59.5 \pm 11.9$ & 54 \\
\hline Hood, $2007^{*}$ & BENDRO 2.5-5mg & Crossover, 51 & $59.5 \pm 11.9$ & 54 \\
\hline Ubaid-Girioli, 2009 & SPIRO $25 \mathrm{mg}$ & Parallel, 39 & $\ldots$ & $\ldots$ \\
\hline Ubaid-Girioli, 2009* & SPIRO 25mg & Parallel, 32 & & \\
\hline Marone, 1985 & CLTD 25-50mg & Single-arm, 10 & $56.7 \pm 2.5(\mathrm{SEM})$ & 40 \\
\hline Weinberger, 2002 & E $50 \mathrm{mg}$ & Parallel, 49 & $\ldots$ & 70 \\
\hline Weinberger, 2002* & E 100mg & Parallel, 43 & $\ldots$ & 61 \\
\hline Weinberger, 2002* & E $400 \mathrm{mg}$ & Parallel, 52 & $\ldots$ & 64 \\
\hline Weinberger, 2002* & E $25 \mathrm{mg}$ BD & Parallel, 51 & $\ldots$ & 73 \\
\hline Weinberger, 2002* & E 50mg BD & Parallel, 49 & $\ldots$ & 70 \\
\hline Weinberger, 2002* & E 200mg BD & Parallel, 43 & $\ldots$ & 69 \\
\hline Weinberger, 2002* & SPIRO 50mgBD & Parallel, 42 & $\ldots$ & 75 \\
\hline Weinberger, 2002* & Placebo & Parallel, 49 & $\ldots$ & 58 \\
\hline Karns, 2012 & E 50mg BD & Parallel, 33 & $56.2 \pm 7.7$ & 57.6 \\
\hline Karns, $2012^{*}$ & Placebo & Parallel, 33 & $59.8 \pm 9.33$ & 66.7 \\
\hline Vasavada, 2003 & TORS 40mg or FURO 80mg & Crossover, 14 & $67 \pm 11$ & 93 \\
\hline Matsui, 2010 & HCTZ $12.5 \mathrm{mg}$ & Parallel, 104 & $68 \pm 9.1$ & 40 \\
\hline Lijnen, 1981 & HCTZ 100mg & Parallel, 5 & $37.2 \pm 2.7$ & 57.1 \\
\hline Lijnen, 1981* & TIE $250 \mathrm{mg}$ & Parallel, 5 & $37.2 \pm 2.7$ & 57.1 \\
\hline Lijnen, 1981* & TIE 1000mg & Parallel, 4 & $37.2 \pm 2.7$ & 57.1 \\
\hline Koenig, 1991 & HCTZ 25mg & Crossover, 51 & $68 \pm 8$ & 20 \\
\hline Saruta, 2004 & Placebo & Parallel, 48 & $54.3 \pm 10.55$ & 68 \\
\hline Saruta, 2004* & $\mathrm{E} 50 \mathrm{mg}$ & Parallel, 48 & $54.2 \pm 11.3$ & 63.3 \\
\hline Saruta, 2004* & E100mg & Parallel, 45 & $52.8 \pm 10.02$ & 69.6 \\
\hline
\end{tabular}




\begin{tabular}{|c|c|c|c|c|}
\hline Study ID, Year & Diuretic, dose & Study design, sample size & Age (years) & Male (\%) \\
\hline Saruta, 2004* & $\mathrm{E} 200 \mathrm{mg}$ & Parallel, 47 & $52.6 \pm 10.76$ & 72.9 \\
\hline Chalmers, 1982 & INDA $2.5 \mathrm{mg}$ & Crossover, 16 & $53 \pm 10$ & 44 \\
\hline Calhoun, 2011 & E 50mg BD & Parallel, 68 & $55.3 \pm 9.1$ & 59.7 \\
\hline Calhoun, 2011* & Placebo & Parallel, 60 & $53.9 \pm 8.7$ & 66.7 \\
\hline Svendsen, 1983 & HCTZ+AMIL & Parallel, 15 & 48.5 & 43 \\
\hline Ubaid-Girioli, 2007 & HCTZ 25mg & Parallel, 18 & $49.3 \pm 7.2$ & 46 \\
\hline Ramsay 1981 & Spiro $25 \mathrm{mg}$ & Crossover, 14 & 50 & 43 \\
\hline Ramsay 1981* & SPIRO 50mg & Crossover, 14 & 50 & 43 \\
\hline Ramsay 1981* & SPIRO 100mg & Crossover, 14 & 50 & 43 \\
\hline Ramsay 1981* & SPIRO $200 \mathrm{mg}$ & Crossover, 14 & 50 & 43 \\
\hline Brummelen, 1979 & HCTZ 50mg BD & Single-arm, 13 & $26-58$ & 100 \\
\hline O'Connor, 1980 & HCTZ & Crossover, 19 & $50.1 \pm 2.6$ & 100 \\
\hline Ferguson, 1982 & HCTZ 50mg & Crossover, 4 & 51 & 66 \\
\hline Matthesen, 2012 & AMIL 5mg BD & Crossover, 23 & $60(45-70)$ & 60.9 \\
\hline Matthesen, 2012* & SPIRO 25mg BD & Crossover, 23 & $60(45-70)$ & 60.9 \\
\hline $\mathrm{Ni}, 2014$ & SPIRO 25mg & Parallel, 40 & $55.7 \pm 12.3$ & 60 \\
\hline $\mathrm{Ni}, 2014^{*}$ & Placebo & Parallel, 36 & $54.9 \pm 14.2$ & 58.3 \\
\hline Koopmans, 1987 & HCTZ 50mg OD & Crossover, 9 & $50.5 \pm 9.1$ & 55.6 \\
\hline Swaminathan, 2008 & SPIRO 25-50mg & Crossover, 33 & 62.6 & $\ldots$ \\
\hline Kreeft, 1983 & CLTD 100mg & Crossover, 19 & $42-66$ & 52.6 \\
\hline Kreeft, $1983^{*}$ & SPIRO 400mg & Crossover, 19 & $42-66$ & 52.6 \\
\hline Jarvis, 2015 & HCTZ 12.5-25mg & Parallel, 10 & $68 \pm 6$ & 50 \\
\hline Yang, 2016 & SPIRO 20-40mg & Parallel, 15 & $44.7 \pm 10.8$ & $\ldots$ \\
\hline Karashima, 2016 & HCTZ $6.25 \mathrm{mg}$ & Parallel, 22 & $65 \pm 1$ & 68 \\
\hline Karashima, 2016* & E $50 \mathrm{mg}$ & Parallel, 23 & $66 \pm 2$ & 68 \\
\hline Karashima, 2016 & SPIRO 12.5-100mg & Parallel, 27 & $56.1 \pm 9.9$ & 40.7 \\
\hline Karashima, 2016* & EPLER 25-100mg & Parallel, 27 & $54.9 \pm 10.7$ & 48.1 \\
\hline Ferrara, 1988 & CLTD $25 \mathrm{mg}$ & Parallel,16 & $49 \pm 8$ & 50 \\
\hline Ferrara, 1988* & Placebo & Parallel, 16 & $49 \pm 8$ & 50 \\
\hline Derosa, 2018 & CAN 50-100mg & Parallel, 81 & $53.4 \pm 7.2$ & 53.4 \\
\hline Derosa, $2018^{*}$ & HCTZ 12.5-25mg & Parallel, 82 & $52.6 \pm 6.9$ & 48.8 \\
\hline Henning, 1980 & $\mathrm{AMIL}+\mathrm{HCTZ}$ & Crossover, 43 & $\ldots$ & 56.4 \\
\hline Krum, 2002 & Placebo (added to ACE) & Parallel, 60 & 54.7 & 51 \\
\hline Krum, 2002* & Placebo (Added to ARB) & Parallel, 58 & 55.1 & 40 \\
\hline Krum, 2002* & EPLER 50-100mg (+ ACE) & Parallel, 63 & 55.7 & 47 \\
\hline Krum, 2002* & EPLER 50-100mg (+ ARB) & Parallel,64 & 54.2 & 49 \\
\hline Derosa, 2016 & CAN 50mg & Parallel, 87 & $57.15 \pm 8.91$ & 65.5 \\
\hline Derosa, $2016^{*}$ & CAN 100mg & Parallel, 88 & $57.75 \pm 9.18$ & 63.6 \\
\hline Belleau, 1982 & HCTZ 50mg & Crossover, 18 & $44(23-58)$ & 54.6 \\
\hline Gerber, 1985 & INDA $2.5 \mathrm{mg}$ & One-arm, 29 & $37 \pm 2$ & 100 \\
\hline Fouassier, 2020 & SPIRO+FUR+AMIL & Parallel, 73 & $53.7 \pm 10.3$ & 79 \\
\hline Dorresteijn, 2013 & HCTZ 25mg & Crossover, 29 & $60(55-63)$ & 74 \\
\hline Parthasarathy, 2011 & SPIRO 75-225mg & Parallel, 61 & $53.2 \pm 10.92$ & 73.2 \\
\hline Parthasarathy, 2011* & E $100-300 \mathrm{mg}$ & Parallel, 67 & $53.9 \pm 10.89$ & 62.9 \\
\hline Solini, 2019 & HCTZ 12.5mg & Parallel, 20 & $62 \pm 8$ & 70 \\
\hline Ohta, 2015 & EPLER 50mg & Crossover, 20 & $71 \pm 13$ & 55 \\
\hline Ohta, $2015^{*}$ & INDA $1 \mathrm{mg}$ & Crossover, 20 & $71 \pm 13$ & 55 \\
\hline Vaclavik, 2014 & Placebo & Parallel, 76 & $59.7 \pm 9.9$ & 63.2 \\
\hline Vaclavik, 2014 & SPIRO $25 \mathrm{mg}$ & Parallel, 74 & $60.4 \pm 9.5$ & 67.6 \\
\hline Kithas, 2010 & HCTZ & Parallel, 21 & $69 \pm 6$ & 52.4 \\
\hline
\end{tabular}




\begin{tabular}{llllll}
\hline Study ID, Year & Diuretic, dose & Study design, sample size & Age (years) & Male (\%) & Ti \\
\hline Kithas, 2010* & SPIRO & Parallel, 24 & $70 \pm 5$ & 58.3 & 24 \\
Grandi, 2002 & CAN 50mg & Parallel, 17 & $\ldots$ & $\ldots$ & 24 \\
Olshan, 1981 & FURO 40mg & Crossover, 12 & $51.6 \pm 2.7$ & 100 & 4 \\
Meier, 1982 & CLTD 100mg/day (responders) & Parallel, 11 & $45 \pm 5$ & 80 & 6 \\
Meier, 1982* & CLTD 100mg/day (non-responders) & Parallel, 9 & $54 \pm 3$ & 80 & 6 \\
\hline
\end{tabular}

TABLE 1. Summary of randomized controlled antihypertensive trials in which plasma aldosterone (PA) was measured before and after treatment with a placebo/diuretic in 1 or more arms or treatment phases. $\mathrm{SPIRO}=$ spironolactone, $\mathrm{HCTZ}=$ hydrochlorothiazide, $\mathrm{CAN}=$ canrenone, $\mathrm{CLTD}=$ chlortalidone, $\mathrm{FURO}$ $=$ furosemide, $\mathrm{INDA}=$ indapamide, $\mathrm{E}=$ Eplerenone, $\mathrm{AMIL}=$ amiloride, $\mathrm{TIE}=$ tienilic acid. ${ }^{*}=$ more than one result in the same study. 
Figure 1.

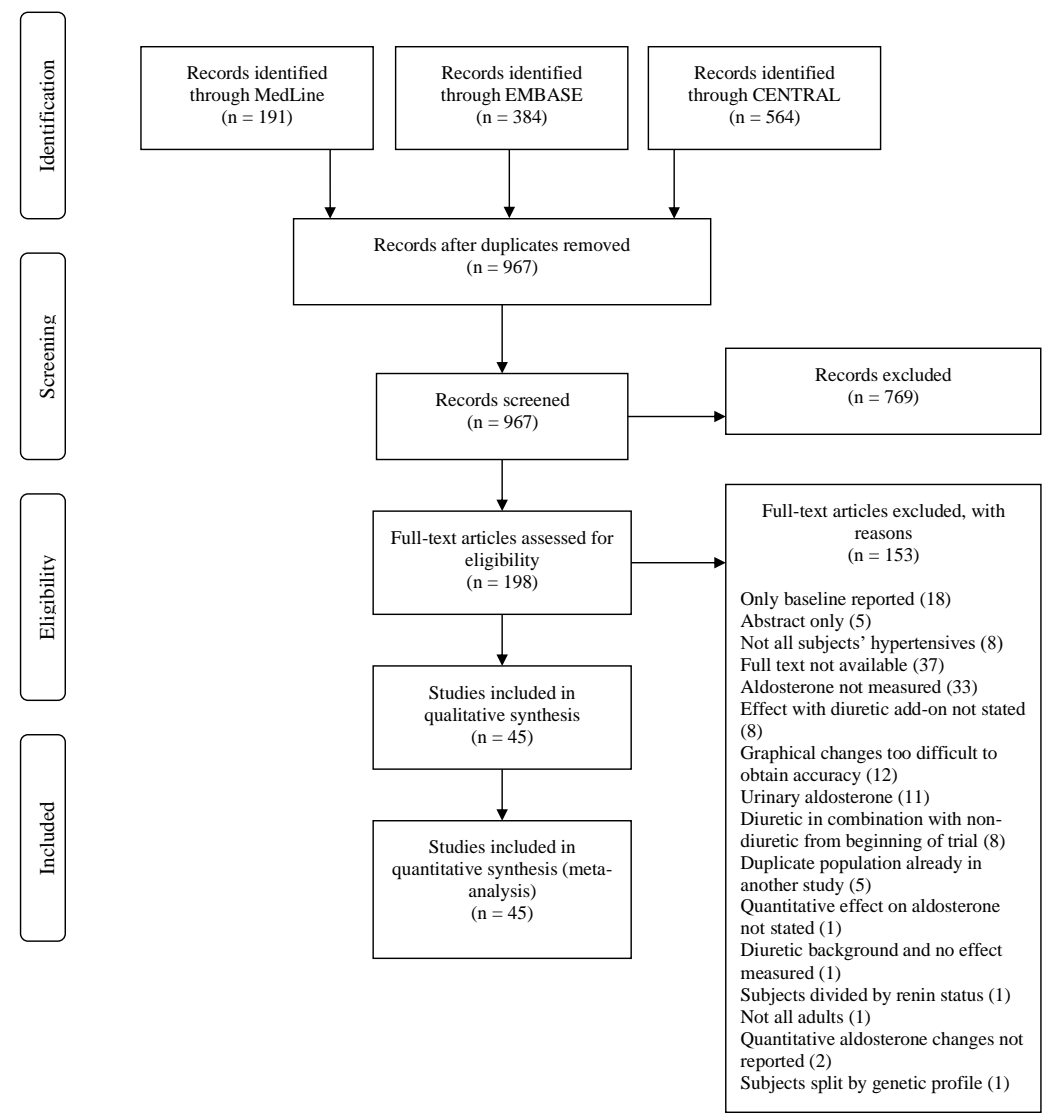




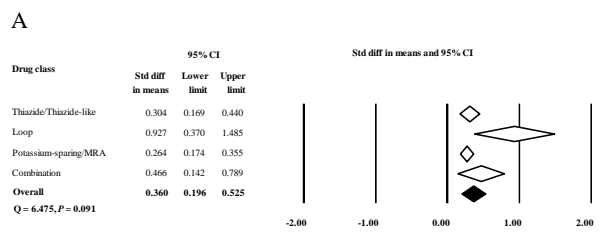

B

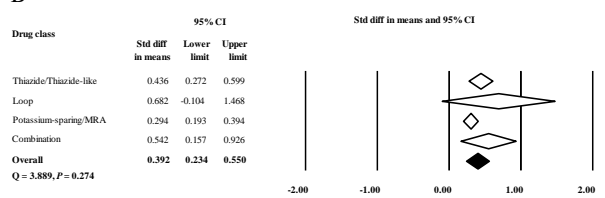

C

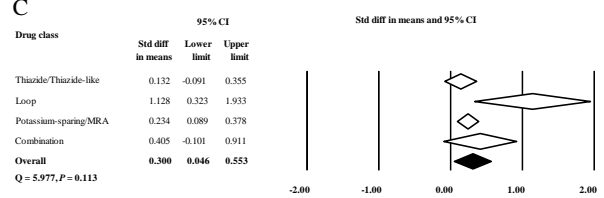

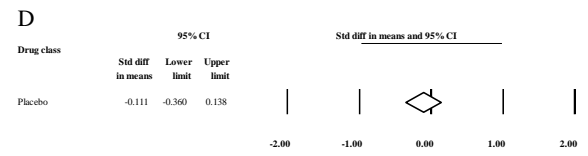


A

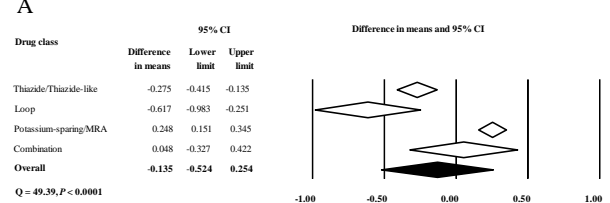

B

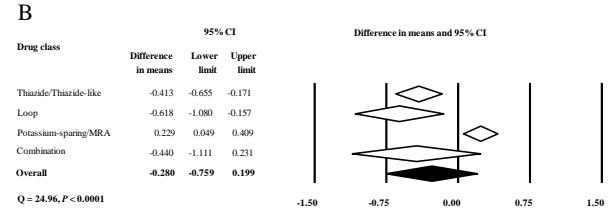

C

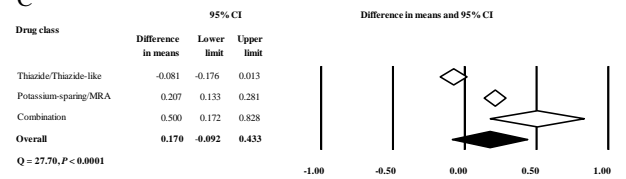

D

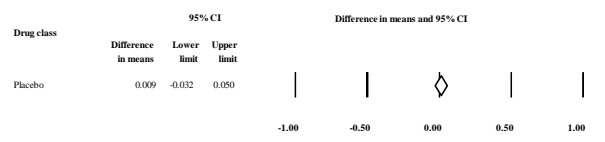


A

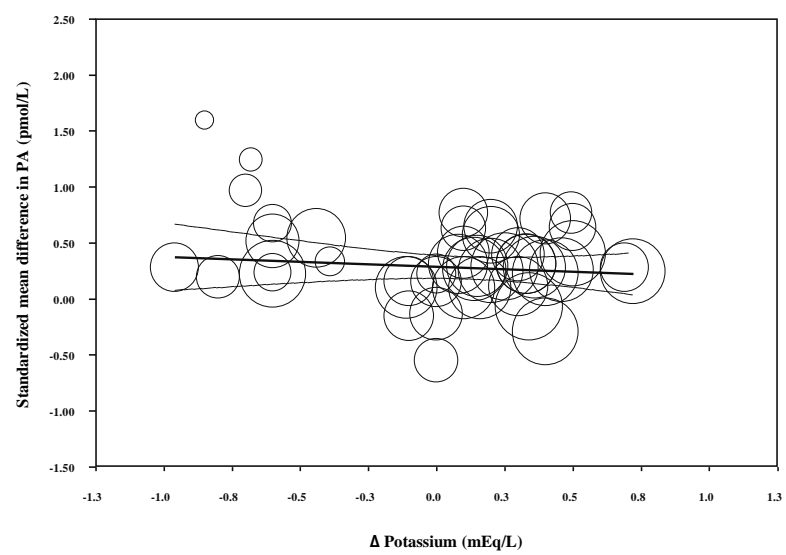

B

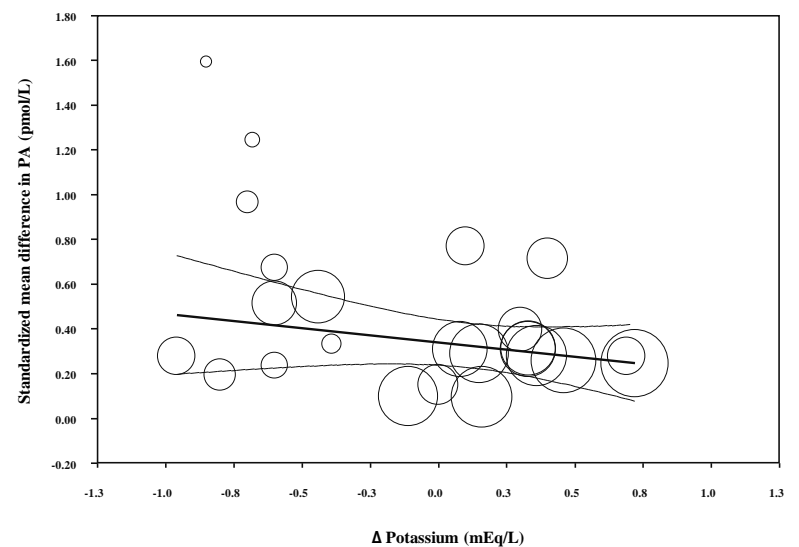


A

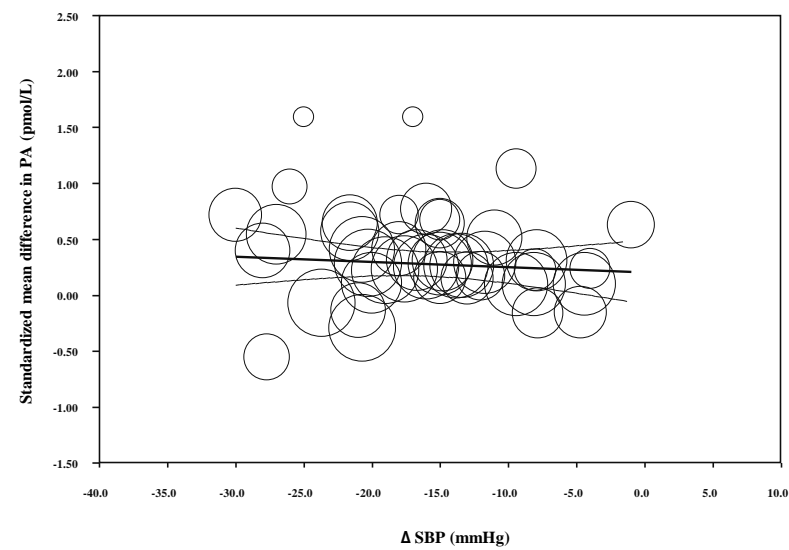

B

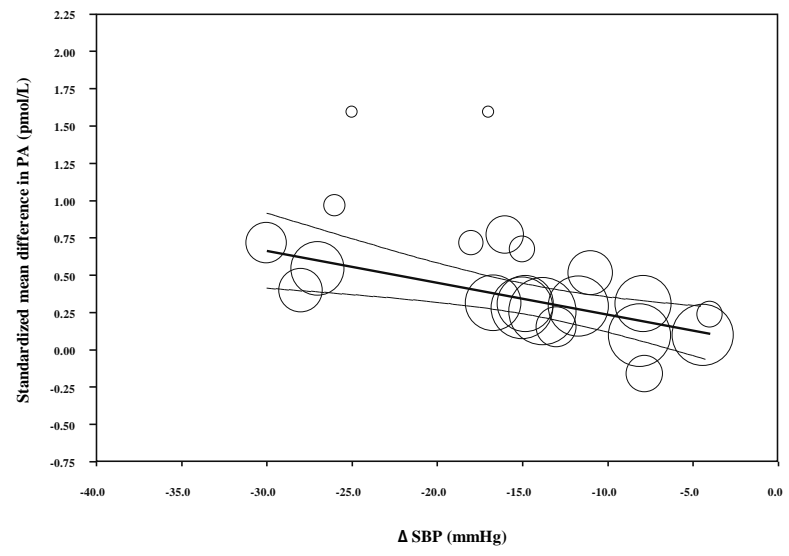




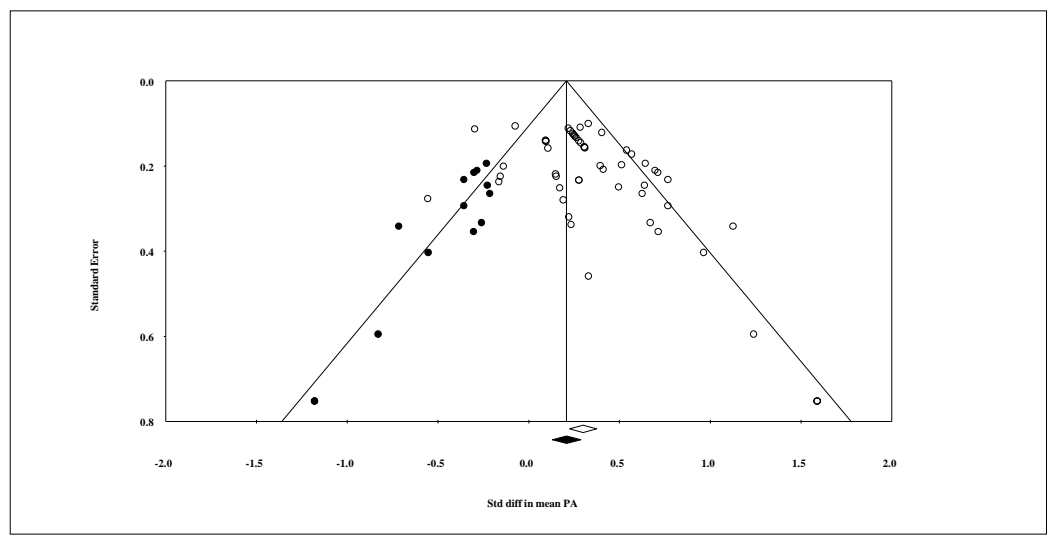

